\title{
A Practical Mandatory Access Control Model for XML Databases
}

\author{
Hong Zhu', Kevin Lï̈, Renchao Jin ${ }^{l}$ \\ ${ }^{1}$ Huazhong University of Science and Technology, Wuhan, Hubei, 430074, P.R. China \\ ${ }^{2}$ Brunel University, Uxbridge, UB8 3PH, UK
}

\begin{abstract}
A practical mandatory access control (MAC) model for XML databases is presented in this paper. The label type and label access policy can be defined according to the requirements of different applications. In order to preserve the integrity of data in XML databases, a constraint between a read-access rule and a write-access rule in label access policy is introduced. Rules for label assignment and propagation are presented to alleviate the workload of label assignments. Furthermore, a solution for resolving conflicts in label assignments is proposed. Rules for update-related operations, rules for exceptional privileges of ordinary users and the administrator are also proposed to preserve the security of operations in XML databases. The MAC model, we proposed in this study, has been implemented in an XML database. Test results demonstrated that our approach provides rational and scalable performance.
\end{abstract}

Keyword: mandatory access control, XML databases, access control model, security. 


\title{
A Practical Mandatory Access Control Model for XML Databases
}

\begin{abstract}
A practical mandatory access control (MAC) model for XML databases is presented in this paper. The label type and label access policy can be defined according to the requirements of different applications. In order to preserve the integrity of data in XML databases, a constraint between a read-access rule and a write-access rule in label access policy is introduced. Rules for label assignment and propagation are presented to alleviate the workload of label assignments. Furthermore, a solution for resolving conflicts in label assignments is proposed. Rules for update-related operations, rules for exceptional privileges of ordinary users and the administrator are also proposed to preserve the security of operations in XML databases. The MAC model, we proposed in this study, has been implemented in an XML database. Test results demonstrated that our approach provides rational and scalable performance.
\end{abstract}

\section{Introduction}

XML has been increasingly used in a variety of applications, and has become the standard for describing and exchanging data over the Internet. As more and more XML documents are stored in XML databases, the security of XML databases has become an important issue. Access control is one of the most important measures in guaranteeing the security of XML databases. The existing access control models for XML databases can be generally catalogued into two types: the discretionary access control model (DAC) and the mandatory access control model (MAC). In the DAC models, a subject who is a creator of an object can discretionally control privileges of other subjects accessing the object in XML databases but cannot resist attacks like Trojan horse because of inherent flaws.

In a MAC model based on BLP model [2], every object is assigned a label which specifies the security privilege of the object, and every user is assigned a label which specifies what objects he/she can access. The label in [2] is a binary-tuple $L=<l, c>$ consisting of a classification $l$, which belongs to an ordered sequence (e.g. unclassified $\leq$ confidential $\leq$ secret $\leq$ top-secret); and a category $c$, which is a subset of a set consisting of non-hierarchical and unordered elements (e.g. \{HumanResource, Financial, Technical $\}$ ). In the following discussion, we assume that $L(s)$ and $L(o)$ denote the labels for a subject and an object respectively. $L(s) . l$ is the classification of the label $L(s)$ and $L(s) . c$ represents the category for the subject $s$. When an object is accessed by a subject, the label of the subject is compared with the label of the object using the following two rules:

(1) simple security property: A subject $s$ can read an object $o$ only if $L(o) \leq L(s)$.

(2) *-property: A subject $s$ can write an object $o$ only if $L(s) \leq L(o)$.

In the simple security property, label $L(o) \leq L(s)$ if and only if $L(o) . l \leq L(s) . l$ and $L(o) . c \subseteq L(s) . c$. We call $L(o) \leq$ $L(s)$ as $L(s)$ dominates $L(o)$. However, when applying the BLP model to databases, the *-property has to be modified to maintain the integrity of the data. Writing objects is allowed only when the subjects and objects have the same label. Therefore, the simple security property and the strict $*_{-}$property need to be enforced $[1,15]$. The strict $*_{-}$ property is described as follows:

strict *-property: A subject $s$ can write an object $o$ only if $L(o)=L(s)$.

The MAC security of a system based on the BLP model is sufficient in many cases, and it has been widely applied in military and government information systems [2]. However, the rules for label comparison in these systems are too rigorous in some cases. It indicates that the larger the category for an object is, the fewer the users that can access it. In some applications $[18,21]$, the rule for label comparison is not as rigorous as this. On the contrary, the requirement is: the larger the category for an object is, the more the users that can access it. Moreover, 
in these applications the structure of the label may be different from the structure of the label in the BLP model. In order to meet the needs of these applications, $[14,18,21]$ have enhanced the flexibility of the MAC mechanism in relational databases. However, the existing MAC models [4, 15, 23] for XML databases are all based on the BLP model. The issue of how to make a flexible and practical MAC model for XML databases has not been addressed in the literatures. In order to overcome the limitations of previous MAC models for XML databases, new rules have to be introduced to make a MAC model be more flexible and can be applied for multi-purposes, meanwhile the security properties of the MAC model are maintained. That is the focus of this study.

We propose a mandatory access control (MAC) model that consists of 12 rules specifying the constraints between a read-access rule and a write-access rule in label access policy, label assignment and propagation, a solution for resolving label assignment conflicts, and updating-related operations (including INSERT, UPDATE and DELETE) for XML databases. Fig. 1 illustrates the relationships amongst these 12 rules in our MAC model. Every node denotes a rule and the edge between two nodes denotes the relationship between the two rules. If there is an edge from rule $A$ to rule $B$, rule $A$ must take effect prior to rule $B$. After the structure of the labels and the label access policy are defined in the MAC, Rule 1 specifies the constraint between the read-access rule and the writeaccess rule in label access policy to preserve the integrity of data in XML databases. Moreover, several label access policies can be defined in an XML database. Different label access policies can be assigned to different XML schemas and XML documents, but one document can only be assigned with one policy. Rule 2 specifies that a XML schema and its documents must be assigned with the same label access policy, and then a subject and an object can be assigned a label respectively. Rule 3 specifies that the label of the root of a schema is the label of its creator. Only after the XML schema is loaded into an XML database, XML documents can then be loaded. Rule 7 indicates that how to specify the label of the root of a XML document that has been loaded. There are three methods of assigning labels to objects in an XML document: assign labels to objects arbitrarily; propagate labels from the ancestors of the objects; and propagate labels from the nodes in a schema associated with the objects. Rule 4 and Rule 5 are related to label propagation. Rule 6 is for deciding the unique label for every object in an XML document. After every element or attribute in an XML document is assigned a label, the update-related operations for the document can be applied, which are specified in Rule 8, Rule 9 and Rule 10. Moreover, the exceptional privileges for ordinary users who create the XML documents and the exceptional privileges for administrator are specified in Rule 11 and Rule 12.

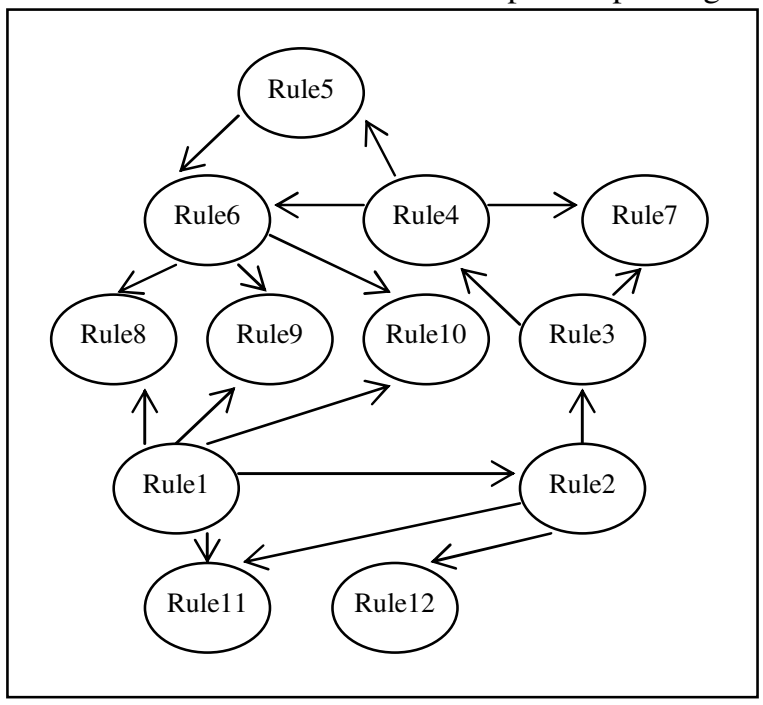

Fig.1. The relationships of rules in our model

The main contributions of this paper are:

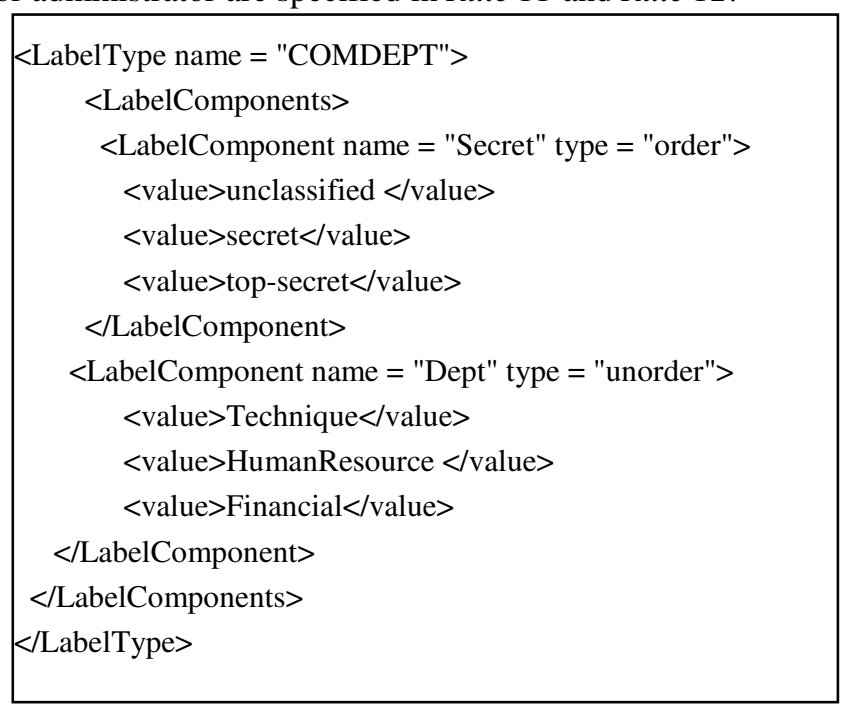

Fig. 2. An example for the label type 
(1) A practical MAC model for XML databases is proposed. The label structure and label access policy can be defined according to the application requirements. We not only can define label access policies which are similar to label access policies in the BLP model for applications with high security, but also can define label access policies according to the application requirements in the way which is different from the BLP model. Therefore, our practical MAC model for XML databases can be used for more different applications.

(2) In order to preserve the integrity of data in XML databases, a constraint between the read-access rule and write-access rule in label access policy has been introduced.

(3) The rules for label assignment and label propagation are addressed to alleviate the workload of label assignments. A solution for the problem of conflicts in label assignments has been proposed.

(4) The rules for update-related operations and the rules for exceptional privileges of both ordinary users and the administrators are developed to preserve the security of operations involving SELECT, UPDATE, and DELETE in XML databases.

(5) We implemented our newly proposed practical MAC model in an XML database. All newly proposed rules and functions have been implemented. This enables us to verify the feasibility, effectiveness and flexibility of our approach. Our approach has been validated against the XMARK benchmark and the test results shown that the performance is rational and scalable.

The rest of this paper is organized as follows: Section 2 illustrates the preliminary concepts of our model. Section 3 describes the practical MAC model for XML databases in detail. Section 4 discusses the implementation considerations of the system. Section 5 illustrates the experimental results. Section 6 describes the related work. Finally, Section 7 presents the conclusions and suggestions for future work.

\section{Preliminary Concepts}

The structure of the label in our MAC model is as follows: each label is specified by a label type, and the label type specifies what components the label type consists of, i.e. every component in a label is specified by a label component type. Normally, the structure of a label is specified by the administrator, which may or may not be the same as the structure of the label in the BLP model.

Definition 1. Label component type. The label component type $L C$ is a set of elements $L C=\left\{c_{1}, c_{2}, \ldots, c_{m}\right\}, m \geq 1$ and it also specifies whether the set is ordered or unordered. If $L C$ is an ordered set, then $c_{1} \leq c_{2} \leq \ldots \leq c_{m}$ and we call it an ordered label component type. Otherwise, we call it an unordered label component type.

Definition 2. Label component. The label component $l c$ is an instance of a label component type. If $L C$ is an ordered label component type then $l c \in L C$, otherwise $l c \subseteq L C$.

Example 1. As shown in Fig.2, two label component types Secret and Dept are defined, where Secret $=L C_{1}=\{$ unclassified, secret, top-secret $\}$ is an ordered label component type with unclassified $\leq$ secret $\leq$ topsecret. Any element in $L C_{1}$ is a label component similar to the classification of the label in the BLP model; Dept $=L C_{2}=\{$ Technique, HumanResource, Financial $\}$ is an unordered label component type, Dept is a set of the department names of a company. Any subset of $L C_{2}$ is a label component similar to the category of the label in the BLP model.

Definition 3. Label type. A label type is an n-tuple $\left.L T=<L C_{1}, \ldots, L C_{n}\right\rangle$, which is composed of $n$ label component types. Here $n \geq 1, L C_{i}$ is a defined label component type. In order to prevent semantic confusion amongst labels, we specify that there is at most one ordered label component type in a label type. If a label type has an ordered label component type, then we specify that $L C_{l}$ must be an ordered label component type. 
Example 2. Using a label type specifies the structure of the label. In Fig.2, a label type COMDEPT $=<$ Secret, Dept $>$ is defined.

Definition 4. Label. A label $L=<l c_{1}, \ldots, l c_{n}>$ is an instance of a label type $L T=<L C_{1}, L C_{2}, \ldots, L C_{n}>$. Where $L C_{i}(1 \leq i \leq n)$ is a defined label component type, and $l c_{i}$ is a label component of $L C_{i}$.

Unlike the label in the BLP model, more than one unordered component type in a label type can be defined in our MAC model according to the various application requirements.

Definition 5. Label access rule. The label access rule is a four-tuple $L A S=(L, T, O P$, type), where $L$ is a set of labels and $T$ is the label type of $L$, type $\in\{r, w\}$ indicates the rule is a read-access rule (type $=r$ ) or a write-access rule $\left(\right.$ type $=w$ ), and $O P$ is a n-tuple $O P=\left\langle o p_{l}, \ldots, o p_{n}>\right.$. For any two labels $l_{l}, l_{2} \in L$, each $o p_{i}$ indicates the operator between $l_{1} \cdot c_{i}$ and $l_{2} \cdot c_{i}(1 \leq i \leq n)$. For an ordered label component type in $T$, the $o p_{i} \in\{E Q, L E, G E, G T, L T\}$ denotes operators in $\{=,<=,>=,>,<$,$\} respectively. For an unordered label component type in T$, the $o p_{i} \in\{I N$, INTERSECTION, CONTAIN, EQUAL\}.

Definition 6. Label access policy. The label access policy is a triple $L P=(S, O, L A S S)$, where $S$ is a set of subjects, $O$ is a set of objects, and LASS is a set of label access rules. The LASS consists of a read-access rule and a write-access rule.

$L P$ indicates that when a subject $s \in S$ reads an object $o \in O$, the labels for the subject and the object must conform to the read-access rule. The labels for the subject and the object are compared according to the operations specified in LP.LASS.OP to determine whether the subject can read the object or not. In the following discussion, if a label $L(s)$ and a label $L(o)$ conform to a read-access rule in $L P . L A S S$, it is denoted as $L(s) \omega_{\text {rin LP.LASS }} L(o)$, if a label $L(s)$ and a label $L(o)$ conform to a write-access rule in $L P . L A S S$, it is denoted as $L(s) \omega_{\text {w in LP.LASS }} L(o)$.

For a label access policy, we can define label access rules in an XML format file. The format of a rule for specifying an operator between two components in the labels of a subject and an object is:

Subject.component-name <operator> Object.component-name

Example 3. Defining a label access policy. In Fig.3, a label access policy for the label type COMDEPT (in Fig.2) is defined. It consists of a read-access rule and a write-access rule.

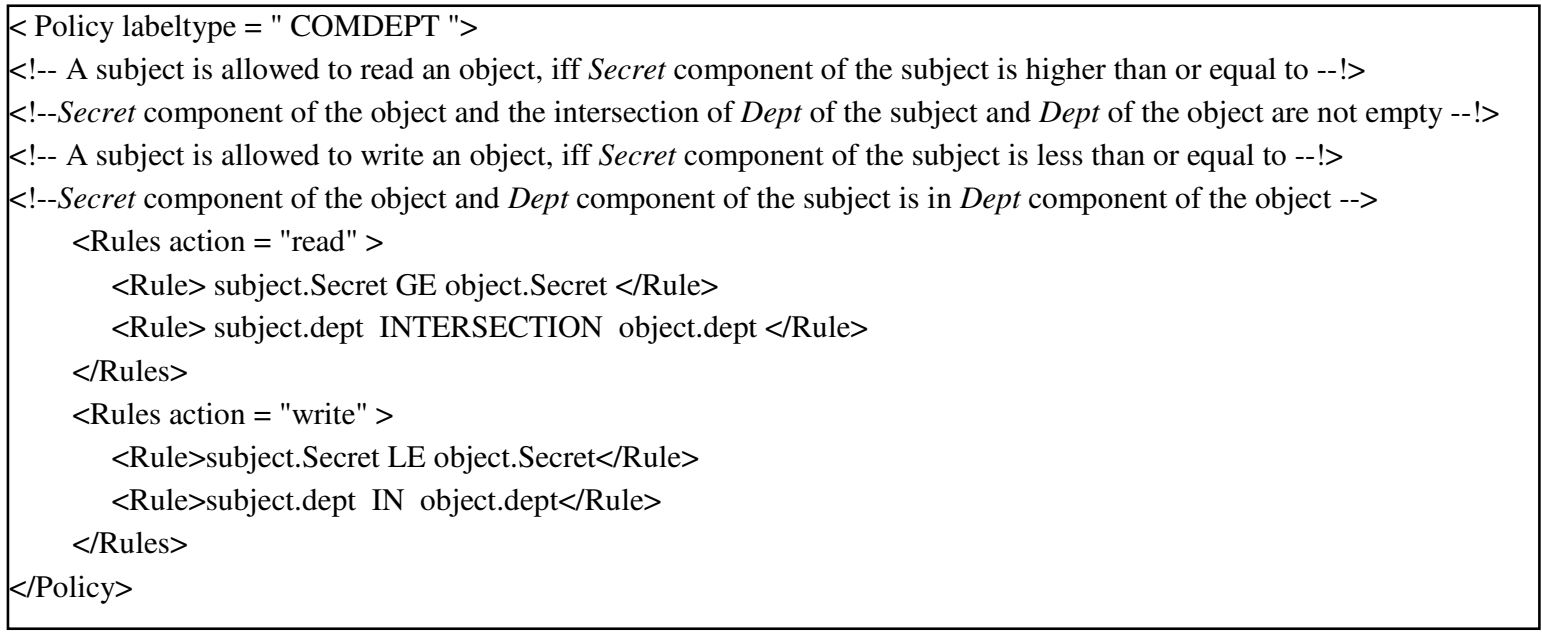

Fig. 3. A label access policy

In XML databases with our mandatory access control, the labels of a subject and an object are compared according to the label access rules. Our model supports the INTERSECTION operator when we compare two labels; this is different from the other MAC models [4, 15, 23] for XML databases. However, when the operation 
INTERSECTION appears in the read-access rule, the domination relationship between two labels has not been defined in our MAC model; therefore, less restriction has been imposed to make it more flexible. This can be demonstrated in the following example.

Example 4. Assume $L_{1}=<$ secret, \{Technique, Financial $\}>$ and $L_{2}=<$ secret, $\{$ HumanResource, Financial $\}>$ are two labels, $L_{1}$ is assigned to a user and $L_{2}$ is assigned to an object. Clearly, the user could read the object under the label access policy shown in Fig. 3 because $L_{1}$ secret $\geq L_{2}$. secret and $L_{1} .\{$ Technique, Financial\} INTERSECTION $L_{2} .\{$ HumanResource, Financial $\}$ is not empty $\left(L_{1}\right.$ and $L_{2}$ matched the read-access rule), meanwhile $L_{2}$. secret $\geq L_{1}$. secret and $L_{2} .\{$ Technique, Financial $\}$ INTERSECTION $L_{1} .\{$ HumanResource, Financial $\}$ is neither not empty ( $L_{2}$ and $L_{1}$ also matched the read-access rule). However, in the BLP model, if $L_{1}$ and $L_{2}$ as well as $L_{2}$ and $L_{l}$ matched the simple security property, then $L_{l}=L_{2}$. Therefore, under the label access policy in Fig.3, for such a case, whether $L_{1}$ dominates $L_{2}$ or $L_{2}$ dominates $L_{1}$ can not be decided. But in our model, the relationship between $L_{1}$ and $L_{2}$ can still exist.

\section{The Mandatory Access Control Model}

\subsection{The rule for label access policy}

When a subject attempts to access an object, the read-access rule is applied to decide whether the user can read the object. When a subject attempts to insert an object into an XML document, or attempts to update or delete an object from an XML document, the read-access rule must be evaluated to locate the object to be inserted, updated or deleted. Therefore, in our MAC model any user can read what he/she wrote before. Otherwise, the integrity of data would not be maintained. Therefore, we introduce the following constraint:

Rule 1. The constraint of label access policy. For a label access policy $L P=(S, O, L A S S)$, the write-access rule contains the read-access rule. Namely, for any subject $s \in S$, and any object $o \in O$, the privilege of $L(s) \omega_{w}$ in LP.LASS $L(o)$ is higher than $L(s) \omega_{\text {rin LP.LASS }} L(o)$.

\subsection{Labelled subjects and XML documents}

The subjects of our MAC model are the users who access the XML databases, or application programs, or agents on behalf of users. After the label type is created, the administrator assigns labels to users, elements or attributes in XML documents or schemas. Every user except the administrator has a unique label, and so does as every object in XML documents.

The objects are elements/attributes in XML documents and schemas. We denote the objects in XML documents using XPath [19]. As the XML document and schema conform to the same XML grammar, the labelled schema and its XML documents have the same notations. We only formally describe the labelled XML document below. The same principle can be applied for formally defining XML schema.

Definition 7. Labelled XML document. The XML document XDoc with labels is an eleven-tuple $X D o c=(V e$, $v r, V a, N s, L s, T, L P$, elemR, attrR, nameR, labelR). We have:

(1) $V e$ is the set of all elements in the document;

(2) $v r$ is the root of the document, $v r$ is also an element of the document, $v r \in V e$;

(3) $\mathrm{Va}$ is the set of all attributes in the document;

(4) Ns is the set of names, including the names of elements and attributes;

(5) $L s$ is the set of all labels with a label type $T$;

(6) $T$ is the label type which specifies the structure of the labels in the document; 
(7) $L P$ is the label access policy, and $L P$ determines the set of label access rules including a read-access rule and a write-access rule;

(8) elem $R$ is a binary-tuple, elem $R \subseteq V e \times V e$. If $e 1 \in V e, e 2 \in V e$, then $(e 1, e 2) \in$ elemR denotes $e 2$ is a subelement of $e 1$ or there exists a link between $e 1$ and $e 2$;

(9) $a t t r R$ is a binary-tuple, $a t t r R \subseteq V e X V a$. If $e \in V e, a \in V a$ then $(e, a) \in \operatorname{attr} R$ denotes $a$ is an attribute of $e$;

(10) name $R$ is a binary-tuple, name $\subset \subseteq N s \times(V a \bigcup V e)$. If $n \in N s, v \in V a \bigcup V e$, then $(n, v) \in$ nameR denotes that $n$ is the name of $v$. As different elements or attributes in the same document may have the same names, one member of $N s$ may be mapped into different members of $\mathrm{Va} \bigcup \mathrm{Ve}$;

(11) labelR is a binary-tuple, labelR $\subseteq(V a \bigcup V e) X_{L s}$. If $L \in L s, v \in V a \bigcup V e$, then $(v, L) \in l a b e l R$ denotes $L$ is the label of $v$, or $L=L(v)$. Different elements or attributes may have the same label, and every element or attribute has only one label.

For a document not being labelled, it is a seven-tuple $D o c=(V e, v r, V a, N s$, elemR, attrR, nameR $)$. The meanings of these symbols are the same as those in the XDoc.

In the following discussion, we use XDoc and XSch to denote a labelled XML document and schema, respectively. For example, we also denote XDoc.T and XDoc.LP as a label type and a label access policy for the XML document. From the definition of XDoc, we have: for any label access rule las $\in$ XDoc.LP.LASS, XDoc.T=las.T.

\subsection{The label assignment rules for XML objects}

Multiple label access policies can be defined for different security requirements but every document can be assigned only one label access policy. After an XML document or schema is loaded, a label access policy is first assigned for them. Then, the labels for elements or attributes in the XML document and schema are assigned.

\subsubsection{The constraint of a label access policy between an XML schema and its documents}

An XML schema defines a set of XML documents with the same structures and similar contents. The label access policy should be the same for the schema and its documents.

Rule 2. The constraint of label access policy between an XML schema and its documents. If the labelled XML schema is XSch and one of its documents is XDoc, then XSch.LP=XDoc.LP.

\subsubsection{The rules for label assignment and propagation}

When an XML schema is created, the label for the root of the schema should be assigned. For an ordinary user, when he/she creates an XML schema, the label for the root of the schema is equal to the label of the user. So we have the following Rule 3:

Rule 3. The label for the root of an XML schema. For an ordinary subject s, if s creates an XML schema sch, then $L($ sch.vr $)=L(s)$.

If the schema is created by the administrator, the label for the root of the schema must be assigned explicitly. Rule 12 (in section 3.3.5) is about the exceptional privilege for the administrator.

There are a large number of elements and attributes in an XML document. If each element or attribute is assigned a label, the workload for management of these labels would be enormous. One solution to his is that we may make use of the following features of XML to alleviate the administrator's workload:

(1) An XML schema defines a set of XML documents with the same structure and similar contents. Usually, these XML documents have the same security attributes;

(2) XML documents are hierarchical and every element has its sub-elements or attributes except for the leaves. 
The administrator needs to only assign labels to XML schemas and some elements in XML documents, then the labels are propagated to instance elements or attributes in the XML documents of the schema, or the labels are propagated to descendent elements and attributes of the labelled elements downward from the root to leaves.

Rule 4. Label propagation from an XML schema to XML documents. For a labelled XML document XDoc and

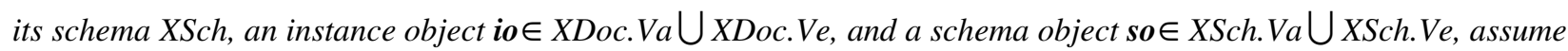
io is one of the instances of the schema object so, then $L($ io $)=L($ so $)$.

Rule 5. Label propagation from an element to its sub-elements and attributes. Assume XDoc is a labelled XML

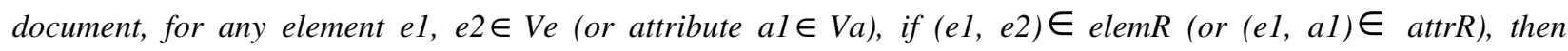
$L(e 2)=L(e 1)($ or $L(a 1)=L(e 1))$.

\subsubsection{A solution for conflicts of label assignments}

Rule 3, Rule 4 and Rule 5 are designed to enhance the flexibility of label assignment and alleviate the workload of the administrator, but may result in the assignments of several different labels to one object. For example, an element in an XML document may have three labels. The first one is propagated from its ancestor, the second one is propagated from the schema, and the third one is assigned directly by the administrator. In order to guarantee that every object in an XML document has only one label, we introduce a rule for deciding labels under a specific label access policy to solve label assignments conflicts.

Rule 6. The label deciding rule. Assume a read access rule lasr $=(L, T, O P, r), L_{1}=<a_{1}, a_{2}, \ldots, a_{n}>\in L_{\text {and }} L_{2}$ $=<b_{1}, b_{2}, \ldots, b_{n}>\in L$ are two labels of label type $T$, if $L_{1}$ and $L_{2}$ are two labels assigned to the same object by direct assignment or by propagation, respectively, we can decide and assign a new label $L_{3}=<c_{1}, c_{2}, \ldots, c_{n}>$ for the object, where each component of $L_{3}$ is decided as follows:

(1) For the ordered label component type in the T, if the operator in the lasr.OP.op ${ }_{1}$ is:

(i) GE or GT, then $L_{3} \cdot c_{1}=\max \left(L_{1} . a_{1}, L_{2} \cdot b_{1}\right)$;

(ii) $L E$ or $L T$, then $L_{3} \cdot c_{1}=\min \left(L_{1} . a_{1}, L_{2} \cdot b_{1}\right)$;

(iii)EQ, then $L_{3} . c_{1}=\max \left(L_{1} . a_{1}, L_{2} \cdot b_{1}\right)$;

Here the $\max \left(l_{1}, l_{2}\right)$ is a function to decide the maximum of $l_{1}$ and $l_{2}, \min \left(l_{1}, l_{2}\right)$ is a function to decide the minimum of $l_{1}$ and $l_{2}$.

(2)In the following formulas, if there is an ordered label component type in the label type then $i \geq 2$, otherwise $i \geq 1$. For an unordered label component type in T, if the operator lasr.OP.op is:

(i) IN, then $L_{3} \cdot c_{i}=L_{1} \cdot c_{i} \cap L_{2} \cdot c_{i}$;

(ii) CONTAIN, then $L_{3} \cdot c_{i}=L_{1} \cdot c_{i} \bigcup L_{2} \cdot c_{i}$;

(iii) INTERSECTION, then $L_{3} \cdot c_{i}=L_{1} \cdot c_{i} \cap L_{2} \cdot c_{i}$;

(iv) EQUAL, then $L_{3} \cdot c_{i}=L_{1} \cdot c_{i}$.

Rule 6 can be extended to decide and assign a unique label for an object when the object may be assigned up to three labels due to direct assignment or propagation. Because the least upper bound does not exist for the INTERSECTION operator in the sense of the least upper bound defined for the CONTAIN operator in [15], a simple deciding rule for an INTERSECTION operator is used in Rule 6. When an INTERSECTION operator is specified for a component in a label access policy, we specify that the component of the resulting label is at the intersection of the corresponding components of two labels. Such a case may occur when some object cannot be accessed by any users except the administrator or the creator of the XML document which the object belongs to. The example below illustrates this problem. 
Example 5. If the labels <secret, \{Technique, Financial $\}>$ and $<$ secret, $\{$ HumanResource $\}>$ are assigned to an object by propagation rules and direct assignment, and the intersection of \{Technique, Financial\} and \{HumanResource\} is empty, then the object cannot be accessed by any user except the administrator and the creator of the XML document which the object belongs to.

Consequently, Rule 6 guarantees that the security of a sub-node is higher than the security of its parent in an XML document. In the following discussion, we use function label_comput $\left(L_{1}, L_{2}\right)$ to denote the result from Rule 6 where $L_{1}, L_{2}$ are two labels. Based on Rule 1 to Rule 6, we have the following property:

Property: For a labelled XML document XDoc and its label access policy XDoc.LP, for elements e1, e2 $\in$ Ve (or attribute $a 2 \in V a$ ), and an ordinary user $s$, if $(e 1, e 2) \in$ elemR (or $(e 1, a 1) \in$ attrR), from Rule1 $\sim$ Rule 6, we have: (1) if $L(s) \omega_{\text {rin XDoc.LP.LASS }} L(e 2)$ (or $\left.L(s) \omega_{\text {rin XDoc.LP.LASS }} L(a 1)\right)$ then $L(s) \omega_{\text {r in XDoc.LP.LASS }} L(e 1)$.

(2) if $L(s) \omega_{\text {win XDoc.LP.LASS }} L(e 2)$ (or $\left.L(s) \omega_{w \text { in XDoc.LP.LASS }} L(a 1)\right)$ then $L(s) \omega_{w \text { in XDoc.LP.LASS }} L(e 1)$.

\subsubsection{The rules for modification-related operations}

As the label access policy for an XML database is defined according to the security requirements of the applications, modification-related operations may have an impact on the labels or the structure of XML documents. When a subject loads an XML document, the label of the subject is compared with the label of the root of the schema. If they conform to the write-access rule in the label access policy, the label of the root would be decided from the label of the subject and the label of the root of the schema of the loaded XML documents by Rule 6. We have the following Rule 7 for loading an XML document:

Rule 7. Loading XML documents. Assume XSch is a labelled XML schema, $s$ is a subject, if $L(s) \omega_{w}$ in XDoc.LP.LASS $L(X S c h . v r)$ and $s$ loads an XML document Doc conforming to XSch, then the loading would be successful and $L($ Doc.vr $)=$ label_comput $(L(s), L(X S c h . v r))$.

Rule 6 and Rule 7 indicate that the MAC can resolve the security problems in the DAC models. We give the following example.

Example 6. Assume that we have only enforced the DAC in an XML database. A user $s 1$ could access several objects (not all objects) in an XML document Docl, but user $s 2$ could not access any objects in Docl. User $s 1$ could leak information in Docl by creating a new document Doc 2 which consists of the objects user $s 1$ could access, and then user $s 1$ authorizes all the access privileges of all objects in $D o c 2$ to $s 2$. Therefore, $s 2$ would indirectly be able to obtain the information in Docl. If the MAC is enforced by the label access policy in Fig. 3 in the XML database, $s 1$ and $s 2$ are assigned labels $L(s 1)$ and $L(s 2)$ respectively $(L(s 1) \neq L(s 2))$, so that $L(s 1) \omega_{\text {rin XDoc.LP.LASS }} L(s 2)$ is true and $L(s 2) \omega_{\text {rin XDoc.LP.LASS }} L(s 1)$ is not true. According to Rule 7, the label of the root in Doc2 would be $L(s 1)$ (assume that there is no schema for Doc2). Although $s 1$ could authorize all the access privileges for objects in $D o c 2$ to $s 2$, according to Rule 6 and Rule 7, all of the labels for objects in Doc2 are equal to $L(s 1)$. So $s 2$ could not access Doc2 because $L(s 2) \omega_{\text {rin XDoc.LP.LASS }} L(s 1)$ would not be true.

If a subject modifies the labels of some objects in an XML document in the update operations, some other users' access privileges may be changed unintentionally. That may cause illegal information transmission (information leaking). So we have the following Rule 8.

Rule 8. Label of the object for update operations. Assume a subject $s$ updates an object $o \in V e \bigcup V a$ in a labelled XDoc, then $L(o)$ is not changed.

For insertion operations, we should first locate the object $o_{1}$ which is the parent of the object $o_{2}$ to be inserted. From the Property, we can also gain access to the parent of $o_{2}$. According to Rule 4 and Rule 5, the inserted object $o_{2}$ may have two labels propagated from its ancestor and schema, respectively. We shall decide $L\left(o_{2}\right)$ and then compare 
the label of the user with $L\left(o_{2}\right)$. If the label of the user and $L\left(o_{2}\right)$ satisfy the write-access rule in the label access policy, the insertion operation is permitted.

Rule 9. Label of the object for insertion operations. Assume user s is going to insert an object $\mathrm{o}_{2}$ as a sub-node of an object $o_{1}$ in a labelled XDoc; $o_{1} \in$ XDoc.Ve and $o_{2} \in$ XDoc.Ve $\bigcup X D o c . V a$. Assume L' $\left(o_{2}\right)$ is the label of $o_{1}$ propagated from the nodes in the schema of XDoc associated with $o_{1}, L\left(o_{2}{ }^{\prime}\right)=$ label_comput $\left(L\left(o_{1}\right), L^{\prime}\left(o_{2}\right)\right)$. If $L(s) \omega_{w}$ in XDoc.LP.LASS $L\left(\mathrm{o}_{2}{ }^{\prime}\right)$, then an insertion operation is permitted, and the final label for $o_{2}: L\left(o_{2}\right)=l a b e l \_c o m p u t(L(s)$, $\left.L\left(\mathrm{O}_{2}{ }^{\prime}\right)\right)$.

As the insertion and deletion operations may modify the structure of the XML documents, the structure of the updated documents must conform to the constraints of their schema. When an XML schema is defined, the maximum or minimum occurrences for the sub-elements of an element may be defined. So the operations should follow the constraints: the number of sub-elements must be no more than the maximum occurrences defined for the element after the sub-element is inserted into an element; the number of sub-elements left must be no less than the minimum occurrences defined for the element after a sub-element is deleted from the element.

Rule 10. Constraints for insertion and deletion operations. XSch is an XML schema, XDoc is one of the documents of XSch, $\left(e_{1}, e_{2}\right) \in X S c h . e l e m R$. Assume $\left(n e, e_{2}\right) \in X D o c . n a m e R$, and there exists the following constraints on $e_{1}$ :

(1)The maximum occurrence of ne is max $_{1}$;

(2)The minimum occurrence of ne is $\min _{l}$;

Then, after any insertion operation on $e 1$, the occurrence of ne $\leq \max _{1}$; after any deletion operation on $e_{1}$, the occurrence of $n e \geq \min _{1}$.

\subsubsection{Exceptional privileges}

Ordinary users can load XML documents and schemas. After an ordinary user has loaded an XML document or a schema and the administrator has assigned the labels, the ordinary user may not be able to query all the objects in the document or schema because of the label access policy. In order to prevent confusions for ordinary users "missing" objects he/she loaded earlier and to prevent transmission of information covertly, we intgioduce exceptional privileges to ordinary users who create XML documents or schemas.

Rule 11. Exceptional privileges for creators of XML documents and schemas. If an ordinary user s loads an $X M L$ document or a schema, and the administrator assigns labels to the objects in the document or the schema, then for any object $o \in V e \bigcup$ Va in XDoc, s can access o without complying with the label access policy.

Although the administrator maintains labels in the system, the administrator does not have a label. How do we deal with the initial labels for the XML documents and schemas loaded by the administrator? To solve the problem, we give the following Rule 12 .

Rule 12. Exceptional privileges for the administrator. The administrator can load XML documents and schemas, and the administrator must also assign an initial label to the root of the loaded XML documents and schemas.

\section{Implementation Considerations}

\subsection{The architecture of the MAC for XML databases}

We have implemented our MAC model in an XML database management system. We used Dom4J as our parser for the XML schemas and documents. The main tool for the program is JDK1.4+Eclipse3.0+Dom4J [6, 7]. The architecture of the XML database management system is shown in Fig.4. It consists of five main modules, which are all within the rectangle with dashed line: policy management module, schema and document loader module, policy 
service module, query module (process read-only operations) and modification module (process update-related operations).

A tool was developed for managing label access policy at the client end. With this tool, the label type and label access policy can be defined and managed. After the policy management module accepts requests from this tool, it converts the label access policy and label type into an XML file and stores the XML file into the security repository. There are two types of XML files in the security repository: label access policy file and label assignment file for users. The security repository is invisible to ordinary users but is visible to the administrator.

The administrator and ordinary users can load the schemas or XML documents by using the schema and document loader module. The initial labels for the schemas and documents are assigned by the policy service module. The labelled XML schemas and documents are stored into the labelled XML documents and schemas repository. Moreover, by using this tool labels are assigned to users and objects in XML documents and schemas. For objects in an XML document, the unique labels are computed and the labelled XML documents and schemas are stored.

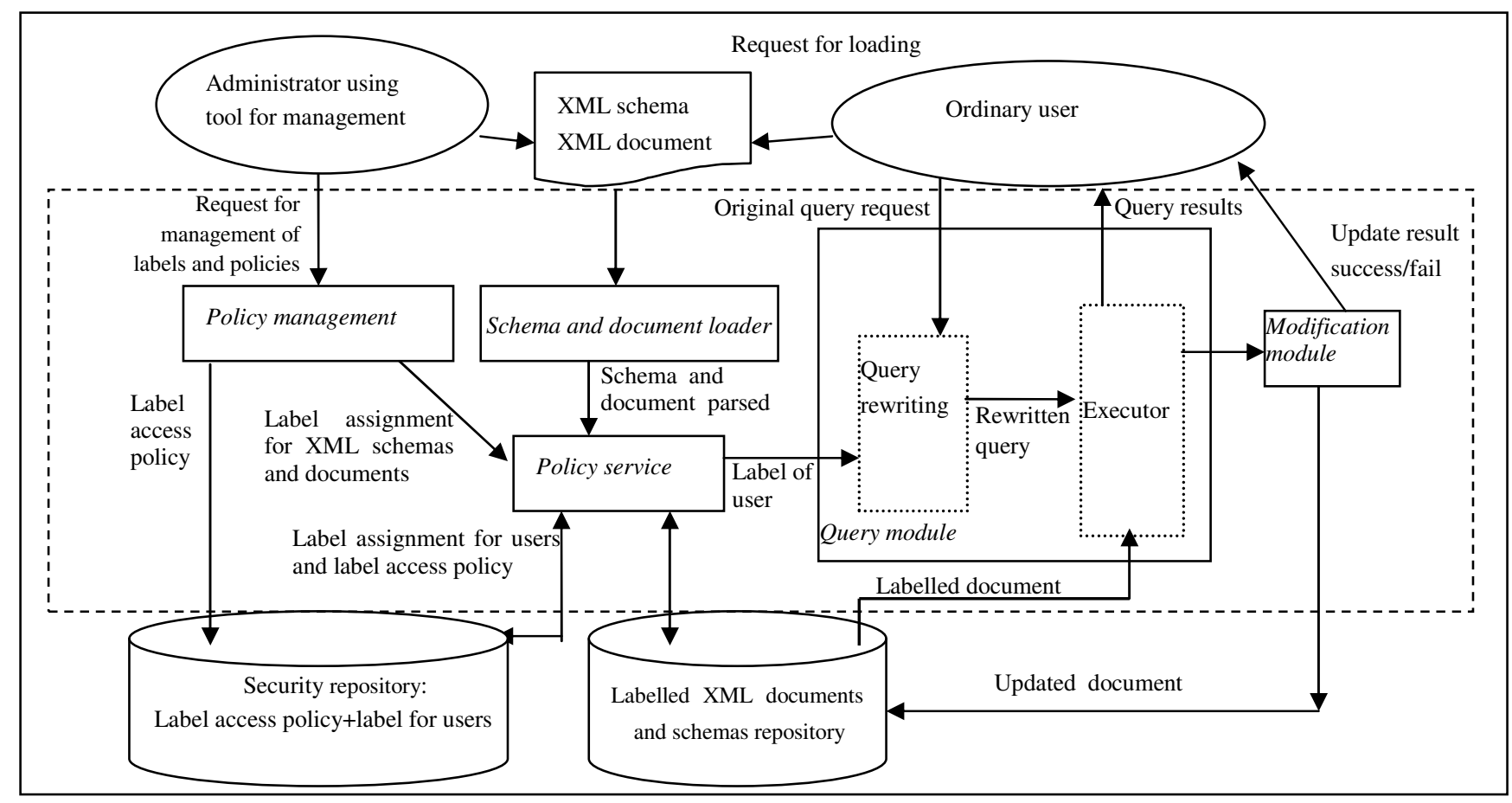

Fig. 4. Architecture of the MAC in XML database

The query module has two sub-modules: query rewriting and executor. When a user submits a query, in the query module the original query is first rewritten according to the label of the user, then the rewritten query is executed, and the final results are returned to the user. The objects in XML documents are updated by the modification module. When a user submits a modification request, the query module is executed first to find or locate the objects to be updated, deleted or inserted, and then updated documents are stored in the XML database.

\subsection{Key techniques for implementation}

\subsubsection{The assignment of labels for users and objects in XML documents}

When a label type is defined, it is converted into an inner denotation in the system. For example, an element in the order label components is denoted as a number, and a set of the unordered label components is represented as a string of ' $I$ ' or ' $O$ ' that denotes whether an element occurs or not. Therefore, if an operator for an unordered label component includes CONTAIN, and when the unordered label component for a user contains the unordered label component for an object, each ' $O$ ' in the string for the unordered label component of the object indicates that the 
character in the corresponding position of the string for the unordered label component of the user should also be ' $O$ '. Similar processes are also developed for IN and INTERSECTION operators. By this way, labels for the users and objects are compared.

In order to assign labels to documents in an XML database, the structure of XML documents is modified. A label is attached as an attribute for each object in the documents. However, the schema of the XML documents should not be changed because the XML schema defines the structure of many XML documents. So when an XML schema is loaded, a schema map document is created for the schema. Initially, the schema map document is the XML schema, then the mapping relationships between the objects in the schema and objects in the XML documents are added, and the labels for the schema are also stored as attributes of the objects in the schema map document. The schema map document is also stored in the labelled XML documents and schemas repository. When the administrator assigns a label to the objects in XML documents or the schemas, Algorithm 1 and Algorithm 2 (shown in Fig.5) are executed to compute the labels for objects in XML documents.

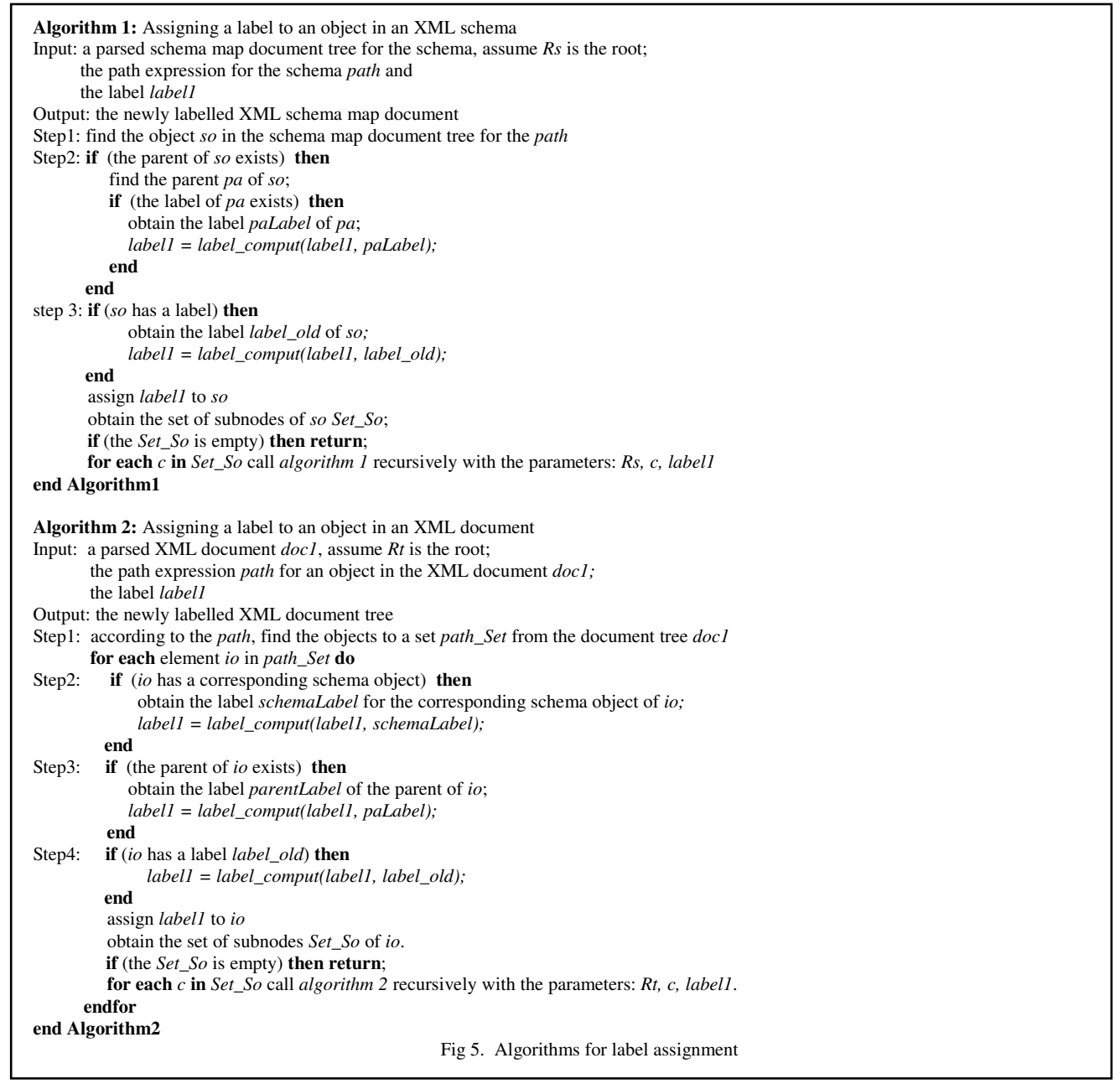

When all the objects in an XML document are assigned a label, the XML document is stored to the labelled XML documents and schemas repository. Clearly, the size of an XML document would be increased and that would affect the performance of the system because of the extra I/O and other operations costs.

\subsubsection{Query rewriting}

After the users and XML documents are labelled, the system could accept the Query operation (deals with readonly operations - SELECT operations) and update-involved operations in the XML database. Because the Query 
operation is an important operation in the system and is the basis of other operations, we describe the process of Query operation in the system in detail.

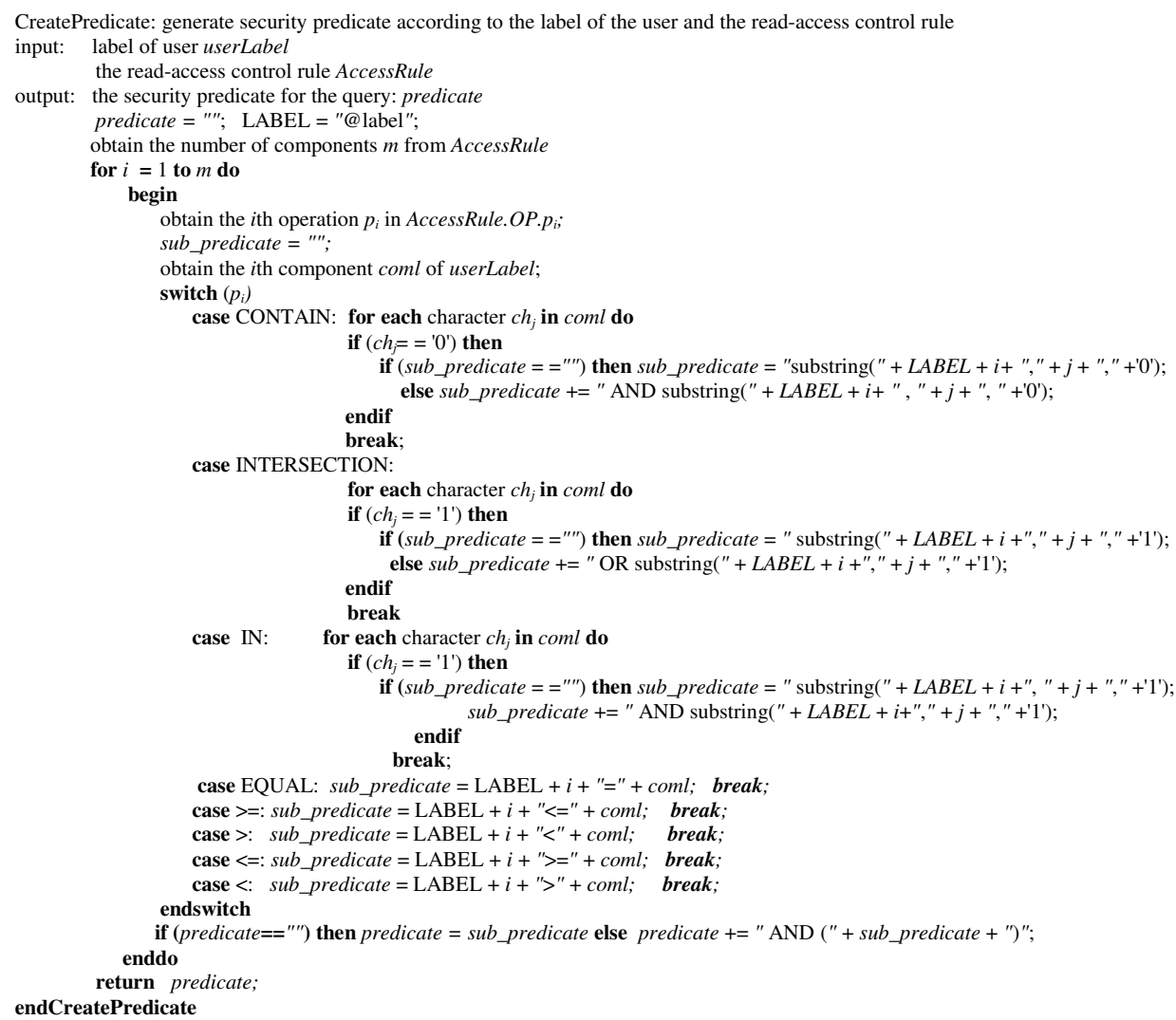


When a user submits a query request, the system checks every XML document involved in the query whether the document was created by the user or not. If it was created by the user, Rule 11 is applied and the query results are returned without the MAC involvement.

If the document was not created by the user, the query processing continues. The label access policy is searched from the security repository to obtain the label access rules. Then the query request is parsed and the query is first rewritten according to the label of the user and read-access rule by adding the label of the user as a searching condition in the query. The pseudo code for the query rewritten is shown in Fig.6. Next, the rewritten query is executed, and the intermediate objects which do not satisfy the read-access rule will be filtered. While a query is processed in this way, an inference attack can be prevented, as illustrated in the following example 7.

Example 7. Assume that the label access rules, as shown in Fig.3, are applied. A user $u$ with label <unclassified, \{Technique\}> in Technical Department submits a query: /companys/employee[salary = "6000"]/name, and the label of object (salary of Alice) is <secret, \{Technique\}>. If the salary of Alice was not filtered out from the results of the query, then a user $u$ can infer that the salary of Alice is 6000. In order to prevent $u$ from inferring unauthorized data by the query, the query above was rewritten by our system as: /companys[@label1<=1 and substring(@label2, 3, 1)='1']/employee[@label1<=1 and substring(@label2,3,1)='1' and salary[@label1<=1 and substring(@label2, 3,1)='1'] = "6000"] / name [@label1 <=1 and substring(@label2, 3, 1)='1']. When the rewritten query is executed, the object salary of Alice is filtered out and the results of the query do not contain salary of Alice.

\section{Experimental Results}

We tested our MAC model in an XML database system for a number of purposes: to demonstrate the validity of our MAC model; to process different types of queries; to evaluate the performance impact of adding our MAC into an XML database. The experimental results are presented in this section. The test environment was two desktop computers with 2.8GHZ CPU, 1G memory and Windows XP (SP2) operating system. The XMark benchmark dataset [26] was used in our experiments. We used the tools of XMark to generate XML documents of different sizes, assigned the labels to objects in XML schemas and documents, and measured the response time of different queries with and without the MAC be installed.

\subsection{The labels for subjects and objects}

We used two types of applications (Appl and App2) in our experiments. The main feature of Appl is that the larger the category for an object is, so a smaller number of users that can access it, and different from Appl, the main feature of App2 is that the larger the set of category for an object is, so a larger number of users can access it. The label types shown in Table 1 are used in the two applications. Two label access policies $A P 1$ and AP2 were defined in the Table 2. From the label access policies in Table 2, we can see that the application Appl is for a multilevel secure system and the application App2 is not.

After the label types and label access policies were created, the administrator loaded the XML schemas and documents, and created four users Lisa, Tom, Alice and Mary for Appl and two users Mary, Tom for App2. The administrator then assigned the labels shown in Table 3 to those users in these two applications. Next, the administrator assigned labels to the XML schemas and documents. Tables 4 and 5 illustrate the labels assigned to XML schema and documents. 
Table 1. The label types

\begin{tabular}{|c|c|c|}
\hline label component & Label component type & The set of elements \\
\hline Level & Ordered component & Common $<$ Private $<$ Secret $<$ Top secret \\
\hline Category & Unordered component & Buyer, Seller, Maker \\
\hline
\end{tabular}

Table 2. The label access policies

\begin{tabular}{|l|c|c|}
\hline $\begin{array}{l}\text { The groups of access } \\
\text { policies }\end{array}$ & Type of access & Label access policy \\
\hline \multirow{2}{*}{$\begin{array}{l}\text { The first label access } \\
\text { policy AP1 for App1 }\end{array}$} & Read rule & $\begin{array}{r}\text { The level of the subject is larger than the level of the object, and the category of } \\
\text { the subject contains the category of the object }\end{array}$ \\
\cline { 2 - 3 } & Write rule & $\begin{array}{r}\text { The level of the subject is equal to the level of the object, and the category of the } \\
\text { subject is equal to the category of the object }\end{array}$ \\
\hline $\begin{array}{l}\text { The second label } \\
\text { access policy AP2 for } \\
\text { App2 }\end{array}$ & Read rule & $\begin{array}{c}\text { The level of the subject is larger than the level of the object, and the category of } \\
\text { the subject is contained in the category of the object }\end{array}$ \\
\cline { 2 - 3 } & Write rule & $\begin{array}{c}\text { The level of the subject is equal to the level of the object, and the category of the } \\
\text { subject is equal to the category of the object }\end{array}$ \\
\hline
\end{tabular}

Table 3. The users in App1 and App2 applications

\begin{tabular}{|l|l|}
\hline Name of users & Label assigned to the user \\
\hline \multicolumn{2}{|c|}{ For App 1} \\
\hline Lisa & $<$ Private, $\{$ Buyer, Seller $\}>$ \\
\hline Tom & $<$ Secret, $\{$ Buyer, Seller, Maker $\}>$ \\
\hline Alice & $<$ Common, $\{$ Buyer $\}$ \\
\hline Mary & $<$ Secret, $\{$ Buyer $\}>$ \\
\hline \multicolumn{2}{|c|}{ For App 2} \\
\hline Tom & $<$ Secret, $\{$ Buyer, Seller, Maker $\}>$ \\
\hline Mary & $<$ Secret, $\{$ Buyer $\}>$ \\
\hline
\end{tabular}

Table 4. Label assignments for XML schema

\begin{tabular}{|l|l|}
\hline \multicolumn{2}{|c|}{ For App1 } \\
\hline Objects in schema & Label \\
\hline /site & $<$ Common, $\{$ Buyer $\}>$ \\
\hline /site/people/person/profile & $<$ Private, $\{$ Buyer, Seller\} $>$ \\
\hline \multicolumn{2}{|c|}{ For App2 } \\
\hline Objects in schema & Label \\
\hline /site & $<$ Common, $\{$ Buyer, Seller, Maker $>$ \\
\hline /site/people/person/profile & $<$ Private, $\{$ Buyer, Seller, Maker $\}$ \\
\hline
\end{tabular}

Table 5. Label assignments for XML documents

\begin{tabular}{|c|c|}
\hline \multicolumn{2}{|c|}{ For App1 } \\
\hline Objects in document & Label \\
\hline //site & $<$ Common, $\{$ Buyer $\}>$ \\
\hline //site/regions/asia/item & $<$ Private, $\{$ Buyer, Seller $\}>$ \\
\hline //site/people & $<$ Private, $\{$ Buyer, Seller $\}>$ \\
\hline //site/people/person/profile/age & $<$ Secret, $\{$ Buyer, Seller, Maker $\}>$ \\
\hline \multicolumn{2}{|c|}{ For App2 } \\
\hline //site/regions/asia/item & $<$ Private, $\{$ Buyer, Seller $\}>$ \\
\hline
\end{tabular}

\subsection{The queries and analysis of their results}

The requests submitted by users in Table 3 are the following four queries:

Q1://site/regions

Q2: //site/people/person/profile[/age= '33']

Q3: //site/regions/*/items/name

Q4: //site/open_auctions/open_auction/initial

Our tests for these queries over different sizes of documents are analogous, in the way that the main difference is that the numbers of nodes contained in the query results are increased when the sizes of the documents are increased. So instead of showing all the results we obtained - due space limit, we only show the results of $Q 2$ for Tom in an XML document with a size of $10.5 \mathrm{MB}$ after the document is labelled, which can be found at Appendix 1 . Furthermore, in Table 6 we list the numbers of nodes returned for different applications, users and queries over XML documents before and after being labelled by our MAC model. 
Table 6. Results of the queries in different applications

\begin{tabular}{|c|c|c|c|c|}
\hline Applications & user & Query & Number of nodes returned with MAC & Number of nodes returned without MAC \\
\hline \multirow{16}{*}{ App1 } & Alice & \multirow{4}{*}{ Q1 } & 31407 & 34650 \\
\hline & Lisa & & 34650 & 34650 \\
\hline & Tom & & 34650 & 34650 \\
\hline & Mary & & 31407 & 34650 \\
\hline & Alice & \multirow{4}{*}{ Q2 } & 0 & 77 \\
\hline & Lisa & & 0 & 77 \\
\hline & Tom & & 77 & 77 \\
\hline & Mary & & 0 & 77 \\
\hline & Alice & \multirow{4}{*}{ Q3 } & 1185 & 1305 \\
\hline & Lisa & & 1305 & 1305 \\
\hline & Tom & & 1305 & 1305 \\
\hline & Mary & & 1185 & 1305 \\
\hline & Alice & \multirow{4}{*}{ Q4 } & 720 & 720 \\
\hline & Lisa & & 720 & 720 \\
\hline & Tom & & 720 & 720 \\
\hline & Mary & & 720 & 720 \\
\hline \multirow[t]{2}{*}{ App2 } & Mary & \multirow[t]{2}{*}{ Q2 } & 77 & 77 \\
\hline & Tom & & 77 & 77 \\
\hline
\end{tabular}

For the application App1, based on Table 4, Table 5, Rule 4, Rule 5 and Rule 6, all the nodes and sub-nodes of the path: //site/regions are labelled with <Common, \{Buyer\}>, except nodes and sub-nodes of the path: //site/regions/asia/item. The nodes and sub-nodes of the path://site/regions/asia/item are labelled with <Common, $\{$ Buyer, Seller\}>. According to the read-access rule of AP1, Alice could not access the nodes and sub-nodes of //site/regions/asia/item because her label is <Common, \{Buyer $\}>$. Therefore, the number of nodes for Alice returned from $Q 1$ is about $10 \%$ less than that of Lisa and Tom, and the number of nodes for Alice returned from $Q 3$ is also about $10 \%$ less than that of Lisa and Tom. Because the nodes and sub-nodes of the path://site/people/person/profile/age are labelled with <Secret, \{Buyer, Seller, Maker\}>, these nodes are only obtained for the query requested by user Tom with the label <Secret, \{Buyer, Seller, Maker\} $>$. Since the label for Mary is $<$ Secret, $\{$ Buyer $\}>$, the label $<$ Secret, $\{$ Buyer $\}>$ and $<$ Common, $\{$ Buyer $\}>$ match the read-access rule in AP1. Therefore Mary can see all the nodes Alice can see. It is exactly what Appl is designed for, and our model can support it effectively.

We can also use our system to support application App2. We only demonstrate the difference of the two applications for the query $Q 2$ because the results for other queries are analogous. According to Rule 4, Rule 5 and Rule 6, the nodes of the path://site/people/person/profile/age are labelled with $<$ Secret, \{Buyer, Seller, Maker $\}$. In application Appl, Mary could not access the nodes labelled with <Secret, \{Buyer, Seller, Maker $\}$, but in Application App2, she could see those nodes. This reflected the purpose of App2. It verified the fact that our system can support such an application which requires that "the larger the category for an object is, so a larger number of users that can access it". More other tests were conducted for various purposes (but due to space limitation, only these two cases are reported here). Based on our tests, we can summery that:

(1) Our MAC model is capable of defining different label access policies according to different application requirements. Tests on two types (Appl and App2) of applications are reported in this paper. Our MAC model can support both of them. They demonstrate that our MAC model is effective and practical.

(2) Our approach provides tools for creating different policies based on different application requirements and produces the results users expected; it can assign labels to XML schemas and documents as well as users for different types of applications. It shows that our approach is very flexible. 


\subsection{The performance of the queries and analysis}

For any operation in XML databases, the introduction of our MAC model would need more time to execute the query request: analyze and rewrite the query, and filter the intermediate results. Nevertheless, the extra time needed for the MAC should not cause a significant decrease in performance. The tests reported in this section are aimed to compare the response time of the system with and without MAC. The SELECT operation is the basis for updateinvolved operations. The cost difference between the read-only operation (SELECT) and update-involved operations can be calculated if the amount of data needed to be updated can be estimated. In most cases, such cost differences are far less than the costs of locating data (SELECT). For simplicity, we only tested SELECT operations in our experiments to measure the overhead of our MAC model. For queries $Q 1 \sim Q 4$, we only show the response time differences for processing queries $Q 1$ over different sizes in our experiments, and because queries $Q 1 \sim Q 4$ are simple queries, the results are similar. In our experiments, the response time of the queries $Q 1 \sim Q 4$ are mainly determined by the sizes of the documents and are not much related to the user who submitted the query. Q5: //listitem//keyword, is a complex query which involves ancestor-descendant structural joins. Fig.7 and Fig.8 show the response time for processing queries $Q 1$ and $Q 5$ on documents of different sizes in the XML database with and without the MAC.

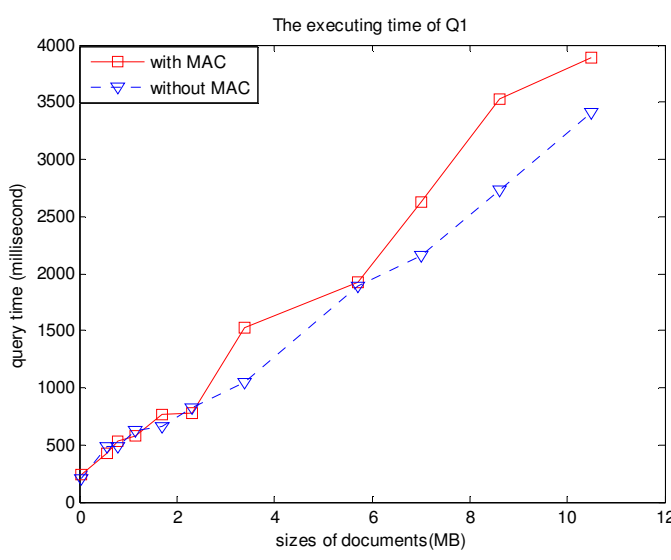

Fig.7 The executing time of Q1

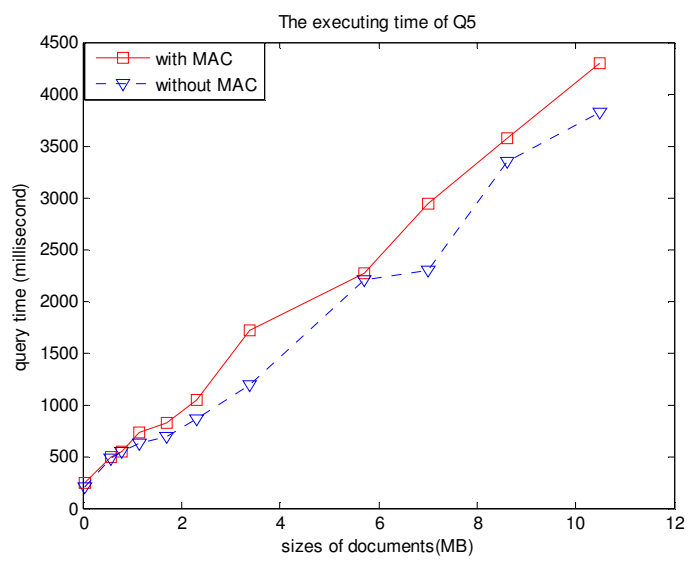

Fig.8 The executing time of Q5

Table 7 The sizes of the documents before and after the documents are labelled

\begin{tabular}{|l|l|l|l|l|l|l|}
\hline Size of documents before labelled & $0.027 \mathrm{MB}$ & $0.56 \mathrm{MB}$ & $1.15 \mathrm{MB}$ & $2.3 \mathrm{MB}$ & $5.7 \mathrm{MB}$ & $7 \mathrm{MB}$ \\
\hline Size of documents after labelled & $0.041 \mathrm{MB}$ & $0.8 \mathrm{MB}$ & $1.7 \mathrm{MB}$ & $3.4 \mathrm{MB}$ & $8.6 \mathrm{MB}$ & $10.5 \mathrm{MB}$ \\
\hline
\end{tabular}

From the figures (Fig. 7 and Fig. 8) and table (Table 7) above, we may observe the following facts:

(1) In our experiments, the sizes of XML documents before and after the documents were labelled are shown in Table 7. We can see that the size of documents increases by about $33 \%$ after the documents are labelled. The performance of the query process decreases due to the extra I/Os are required when the same queries are executed on the same documents after the documents are labelled (see Fig. 7 and Fig.8). When the sizes of documents are small, there is not much difference on response time for labelled and unlabelled documents, but when the size of documents increases the difference on response time can be clearly observed.

(2) When $Q 1$ and $Q 5$ are executed, one extra cost when MAC installed is filtering the intermediate results to obtain the final results (in addition to the cost of extra disk $\mathrm{I} / \mathrm{O}$ access due to the documents being labelled and documents sizes have been increased). When the size of the documents is increased, the performance of the system decreases, but the processing time for the same query over the same document before and after the document being 
labelled does not increase significantly, no matter the queries are simple or complex. As a result, it can be seen that the overall MAC system performance is rational, scalable and acceptable.

\section{Related Work}

A number of measures have been proposed for XML database security, including access control, encryption [9], secure XML information publishing [13], digital signatures and more, but it is generally agreed that access control is the most effective approach. There are usually three types of access control: mandatory access control (MAC), discretionary access control (DAC) and role-based access control (RBAC) [17, 25].

Based on the BLP model [2], SungRan Cho et al [4] proposed the first MAC model for XML documents. However, in the model [4] the structure of DTD needs to be modified, and the structure of XML documents also has to be changed. Dong-Zhan Zhang et al [23] presented an extended MAC model for XML. They used strategies analogous to E. Damiani's model [5] for authorizing privileges and resolving label assignment conflicts but they defined their own label assignment and overriding rules. Lan $\mathrm{Li}$ et al [15] proposed a MAC model for XML documents, in which labels can be assigned to users and elements/attributes in XML documents. When a user wants to access an object in an XML document, the label of the user and the label of the element/attribute are compared according to the simple security property and the strict *-property in the BLP model. Since these three models are all based on the BLP model, they inherited its drawbacks - lack of sufficient flexible mechanisms to define different security policies for different applications. Different from those models, our MAC model can define the label structures and label access policies according to the security requirements of XML applications. Not only we can define label access policies for XML database systems in the similar ways that models [4, 15, 23] can, but our model also can work for multi-purpose applications. Meanwhile constraints between a read-access rule and a write-access rule are maintained so that the integrity of data is preserved. In addition, the rules for update-related operations, for exceptional privileges of both ordinary users and the administrators are proposed to maintain the security of the XML documents.

For relational database systems, Walid Rjaibi [18] proposed a multi-purpose MAC policy to enhance the flexibility of the MAC model. The administrator can define label types, label access rules, and exceptional privileges. This policy is suitable for different requirements and applications in relational databases. In addition, Oracle 10g [14, 21] provides a label security product to enhance the flexibility of MAC. The models for Oracle databases are only suitable for relational databases, and in the model [18], the domination relationship between two labels is not discussed in detail. Inspired by the MAC policy in [18], a MAC model for XML databases is proposed. Meanwhile, the domination relationship in our model is not determined for any two labels because of the need of processing the INTERSECTION operation for label comparison (the reason was explained in Example 4 in Section 2).

Another type of XML access control is based on DAC [3, 5, 8, 11, 12, 16, 24]. However, these models cannot resist attacks like Trojan horse that may result in information leaks. The reason was explained in Example 6 (in Section 3).

XACML [17] is a specification of OASIS to express and deploy an access control policy based on XML and it is combined with RBAC models for XML [8, 25]. Although RBAC models are used in many applications, they can simplify the management of privileges of different roles for users. However, the limitation of the RBAC for XML has analogous security problems in DAC models because the invisible and sensitive data for a user or a role could be leaked by users or roles through transferring their own privileges to the other users or the roles (see Example 6). In contrast, our model can prevent such security problems which exist in DAC and RBAC models. 


\section{Conclusions}

Providing mandatory access control model for databases is important for many database applications. In this study, we have developed a practical MAC model for XML databases for different purposes, including multilevel secure XML database systems. In our MAC model, label access policies can be defined according to the requirements of various applications, which enhance the flexibility of MAC models in general. A constraint between a read-access rule and a write-access rule has been proposed to maintain the integrity of data. Rules for label assignment and propagation are presented to alleviate the workload of label assignments, and a rule for solving label assignment conflicts is also introduced. Moreover, rules for modification-related operations, and exceptional privilege rules for ordinary users and the administrators are introduced. Nevertheless, the proposed MAC policy can be flexibly used in XML databases; therefore, it can be regarded as a generalization of BLP model for XML.

Our MAC model has been implemented in an XML database, and the experiments demonstrate the effectiveness of our approach; our approach can define different access policies, and is able to achieve and realize the design purposes of different access policies. A performance study on the overhead caused by introducing the MAC on an XML database was also carried out; the test results indicate that the performance is rational, scalable and acceptable overall.

In the future, we plan to extend out model to resolve the polyinstantiation problem for modification operations. We are also studying the issues on optimizing system performance.

\section{Acknowledgements}

The work presented in this paper is supported by 863 hi-tech research and development program of China, the granted number: 2006AA01Z430.

\section{References}

[1] V. Atluri, S. Jajodia, E. Bertino, Alternative correctness criteria for concurrent execution of transactions in multilevel secure databases, IEEE Transactions on Knowledge and Data Engineering, 1996, 8(5):839 - 854.

[2] D.E. Bell and L.J. LaPadula, "Secure Computer Systems: Unified Exposition and Multics Interpretation," Technical Report MTR-2997, The Mitre Corp., Bedford, Mass., 1976.

[3] E. Bertino, E. Ferrari, Secure and Selective Dissemination of XML Documents, ACM Transactions on Information and System Security, 2002 , 5(3):290 331.

[4] S.R. Cho, S. Amer-Yahia, L. VS Lakshmanan, and D. Srivastava, Optimizing the Secure Evaluation Of Twig Queries. Int. Conference On VLDB, 2002.

[5] E. Damiani, V. SDC, S. Paraboschi, P.Samarati . A fine-grained access control system for XML documents. ACM Transactions on Information and System Security, 2002 , 5(2):169 202.

[6] Dom4J: the flexible XML framework for Java, http://www.dom4j.org/.

[7] Eclipse Downloads, http://www.eclipse.org/downloads/.

[8] W.F. Fan, C.Y. Chan, M. Garofalakis, Secure XML Querying with Security View. ACM SIGMOD 2004.

[9] J.Gao, T.J. Wang, D.Q. Yang, XFlat: Query-friendly encrypted XML view publishing, Information Sciences, 2008, 178, 774787.

[10] H. He, R. Wong. A Role-Based Access Control Model For XML Repositories. in:Qing Li, Z.Meral Ozsoyoglu, Roland Wagner eds.. Proc of WISE 2000. Hong Kong,China. June, 2000. Hong Kong,China: IEEE Computer Society, $2000.138 \sim 145$.

[11] M. Iwahara, R. Hayashi, S. Chatvichenchai, C. Anutariya and V. Wuwongse, Relevancy-Based Access Control and Its Evaluation on Versioned XML Documents, ACM Transactions on Information and System Security, Vol. 10, No. 1, Feb. 2007, Pages 1 31. 
[12] Y. Kanza, A. O. Mendelzon, R.J. Miller, and Z. Zhang, Authorization-Transparent Access Control for XML under the NonTruman Model, EDBT 2006.

[13] H.K. Ko, M.J. Kim, S.K. Lee, On the efficiency of secure XML broadcasting, Information Sciences, 2007, 5505-5521.

[14] J.E. Levinger. Oracle ${ }^{\circledR}$ Label Security Administrator's Guide10g Release 1 (10.1) Part No. B10774-01, December 2003.

[15] L. Li, X. Jiang, and J.H. Li, Enforce Mandatory Access Control Policy on XML Documents, in Proceedings in 7th International Conference of Information and Communications Security:, ICICS 2005, Beijing, China, December 10-13, 2005.

[16] M. Murata, A. Tozawa, M. Kudo,and S. Hada, XML Access Control Using Static Analysis, ACM Transactions on Information and System Security, Vol.9, No.3, Aug.2006, Pages 292-324.

[17] OASIS standard: eXtensible Access Control Markup Language (XACML) Version 1.0. http://www.oasis-open.org /committees /xacml/ repository /oasis-xacml-1.0.pdf, 18 February 2003.

[18] W. Rjaibi, P. Bird. A Multi-Purpose Implementation of Mandatory Access Control in Relational Database Management Systems. Proceedings of the 30th VLDB Conference, Toronto, Canada, 2004.

[19] XML Path Language (XPath) 2.0. W3C Recommendation 23 Jan. 2007, http://www.w3.org/TR/xpath20/.

[20] J.P. Yoon, Authorization: A Bitmap Indexing Scheme for High-Speed Access Control to XML Documents, IEEE Transactions on Knowledge and Data Engineering, Vol.18, No.7, July 2006, pages 971-987.

[21] X.D. Yuan, Y.Feng, The Model of Mandatory Access Control with Extended Security Label, Chinese Journal of Computers, October 2000: 1096 1100.

[22] T. Yu, D. Srivastava, L. V. Lakshmanan, and H. V. Jagadish. Compressed Accessibility Map: Efficient Access Control for XML. In Proc. 28th Int. Conf. on Very Large Data Bases, pages 478-489, 2002.

[23] D.Z. Zhang, Y.S. Xue, An Extended Mandatory Access Control Model for XML, in Proceedings Advances in Computer Science - ASIAN 2005: 10th Asian Computing Science Conference, Kunming, China, December 7-9, 2005.

[24] H.X. Zhang, N. Zhang, K. Salem, D.H. Zhuo, Compact access control labeling for efficient secure XML query evaluation, Data \& Knowledge Engineering, 2007, 60, 326-344.

[25] X.W. Zhang, J. Park and R. Sandhu, Schema based XML Security: RBAC Approach, Technical Report, IFIP WG 11.3, 2003.

[26] The XML benchmark project. Available from: <http://www.xml-benchmark.org>.

Appendix 1: The results of $Q 2$ for Tom over an XML document of 10.5MB (after the document is labelled). There are 77 nodes in the results.

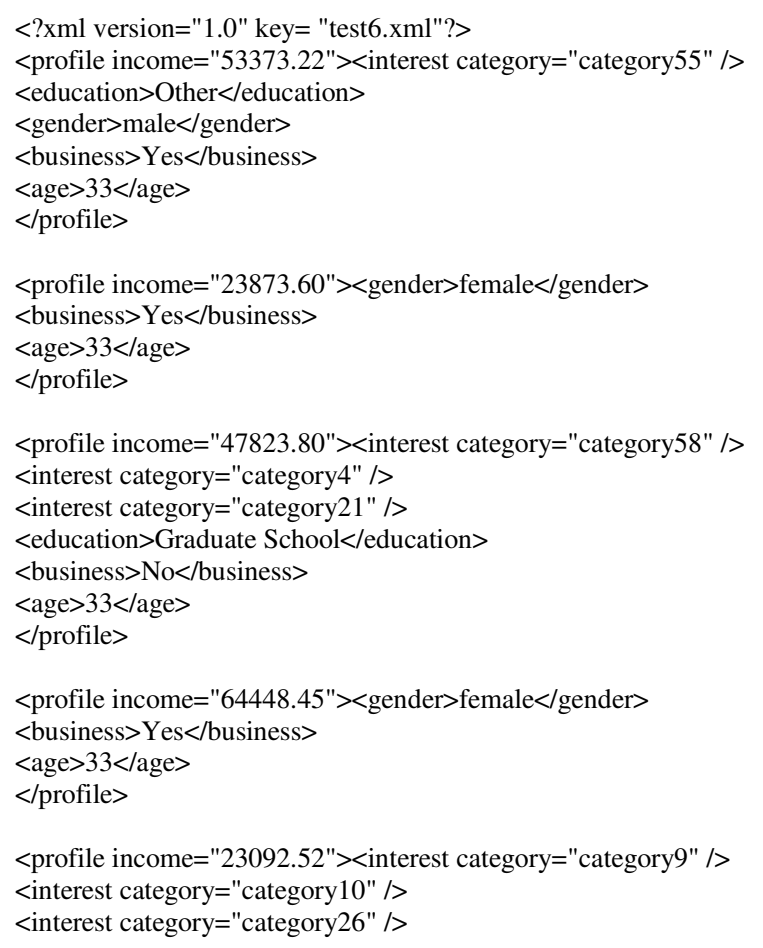


$<$ interest category="category51" />

$<$ education $>$ Graduate School</education $>$

$<$ business $>$ Yes $<$ /business $>$

$<$ age $>33<$ age $>$

$</$ profile $>$

$<$ profile income="43900.78" ><interest category="category56" / >

$<$ interest category="category57" />

$<$ education $>$ High School</education $>$

$<$ business $>$ Yes $<$ /business $>$

$<$ age $>33<$ age $>$

$</$ profile $>$

$<$ profile income="65935.40" $><$ interest category="category40" / > $<$ interest category="category30" / >

$<$ gender $>$ male $<$ /gender $>$

$<$ business $>$ No $<$ /business $>$

$<$ age $>33<$ /age $>$

$</$ profile $>$

$<$ profile income="62004.90" $><$ interest category="category37" / > $<$ gender $>$ male $<$ /gender $>$

$<$ business $>$ Yes $<$ /business $>$

$<$ age $>33<$ /age $>$

$</$ profile $>$

$<$ profile income="9876.00" $><$ interest category="category30" $\mid>$

$<$ education $>$ Graduate School</education $>$

$<$ business $>$ Yes $<$ /business $>$

$<$ age $>33<$ /age $>$

$</$ profile $>$

$<$ profile income="31709.85"><interest category="category50" /> $<$ interest category="category $46 " />$

$<$ interest category="category31" / >

$<$ education $>$ Other $<$ /education $>$

$<$ business $>$ No $<$ /business $>$

$<$ age $>33<$ /age $>$

$</$ profile $>$

$<$ profile income="13560.28" ><interest category="category29" />

<interest category="category3" />

$<$ interest category="category $40 "$ / >

$<$ interest category="category29" />

$<$ interest category="category $27 " />$

$<$ interest category="category $18 " />$

$<$ interest category="category10" />

$<$ education $>$ Other $<$ /education $>$

$<$ business $>$ Yes $<$ /business $>$

$<$ age $>33<$ /age $>$

$</$ profile $>$

$<$ profile income="52458.60" ><interest category="category40" />

$<$ interest category="category $20 " />$

$<$ interest category="category16" />

$<$ interest category="category $44 "$ / >

$<$ interest category="category $48 "$ / $>$

$<$ business $>$ No $</$ business $>$

$<$ age $>33<$ /age $>$

$</$ profile $>$ 